\title{
A double blind, randomised, controlled trial of glutamine supplementation in parenteral nutrition
} J Powell-Tuck, C P Jamieson, G E A Bettany, O Obeid, H V Fawcett, C Archer, D L
Murphy
Rank Department of Human Nutrition, St Bartholomew's and the Royal London Hospital School of Medicine and Dentistry, London, UK

J Powell-Tuck

C P Jamieson

G E A Bettany

O Obeid

H V Fawcett

C Archer

Pharmacy, the Royal London Hospital, Whitechapel, London, UK

D L Murphy

Correspondence to: Dr J Powell-Tuck, Rank Department of Human Nutrition, St Bartholomew's and the Royal London Hospital School of Medicine and Dentistry, Whitechapel, London E1 1BB, UK.

Accepted for publication 2 February 1999

\begin{abstract}
Background and aims-To determine whether the inclusion of $20 \mathrm{~g}$ free glutamine as part of the nitrogen source of parenteral feeds reduces length of hospital stay or mortality.

Methods-In a randomised, double blind, controlled trial in 168 patients clinically accepted for parenteral nutrition, standard feeds were compared with feeds in which $3.8 \mathrm{~g}$ of the total nitrogen was replaced with the equivalent $20 \mathrm{~g}$ glutamine. A minimum of $11 \mathrm{~g}$ nitrogen/ day was used in all patients. Daily intakes of energy and nitrogen were determined using a validated computer protocol and were similar for the two groups. All feeds included trace elements, vitamins, electrolytes, and minerals.

Results-A total of 85 patients received a median of eight (interquartile range 5-13) daily feeds containing glutamine while 83 received a median of eight (5-15) standard feeds. No difference between groups was detected for infective complications. Twenty control patients and 14 who had received glutamine died during their hospital stay (NS). Median length of stay was 32 (23-52) days on glutamine, which was not significantly different from the control value of 35 (25-55) days. Glutamine was associated with a significant $(p<0.03)$ reduction in length of stay in surgical patients (45 days (range 29-81) versus 30 days (range 19-54)).

Conclusion-The benefit from glutamine supplementation of parenteral feeds as used in this trial has not been proved. Supplementation may have advantages in surgical patients and in haematological malignancy. Further trials are required. (Gut 1999;45:82-88)
\end{abstract}

Keywords: glutamine supplementation; parenteral nutrition

Though glutamine is readily synthesised by body tissues, free glutamine and plasma concentrations are diminished in metabolic stress, and standard parenteral feeding regimens do not supply it because of problems with stability and solubility. ${ }^{1}$ A summary ${ }^{2}$ of previous publications has drawn attention to the possibility that glutamine supplementation of parenteral nutrition solutions might be beneficial, principally by improving lymphocyte function $^{34}$ and by preventing intestinal mucosal atrophy. ${ }^{3-7} \mathrm{~A}$ recent study in trauma patients has suggested that glutamine supplementation of enteral feeds can reduce infective complications. $^{8}$

At the Royal London Hospital all adult parenteral nutrition is prescribed and supervised by a specialist multidisciplinary team. It is reserved for those who cannot be fed enterally; the patients fed parenterally are often critically ill, as indicated by a prospective audit of our practice between May 1992 and December 1993 which showed an overall mortality of 35\% among 192 parenterally fed patients over this 20 month period. Median (interquartile range) duration of parenteral feeding was seven (4-16.5) days; median length of stay was 36 (22-55) days.

Studies in patients undergoing treatment for haematological malignancies have suggested that glutamine supplementation might be beneficial, ${ }^{9}{ }^{10}$ and a controlled trial in the context of an intensive care unit has suggested long term benefits in mortality. ${ }^{11}$ We wanted to know whether the routine use of glutamine supplementation in our parenteral feeds could reduce overall mortality and morbidity. This paper describes a clinical trial designed to examine this question, and puts the trial results into the context of the pretrial audit.

\section{Methods}

The trial was approved by the ethics committee of the Royal London Hospital.

PROTOCOL

This was a randomised, double blind trial of the inclusion of $20 \mathrm{~g}$ glutamine into complete parenteral feeds versus standard feeds (complete except that, as is presently routine, no glutamine was added). The feeds were continued until the patient no longer required parenteral feeding as judged clinically.

\section{Patients included}

Patients included were those consenting adults, aged 18-85 years, referred to the nutrition team, for whom parenteral feeding was considered to be clinically indicated. This decision, though subjective, was based on the patient's Quetelet index, percentage weight loss, our perception of the likelihood of achieving adequate enteral nutrient intake soon, and the patient's state of catabolism.

\section{Patients excluded}

Patients were excluded if they did not consent, if the 24 hour infusion needed to be restricted

Abbreviations used in this paper: $\mathrm{N}$, nitrogen; $\mathrm{RNI}$, reference nutrient intake. 
to less than 2 litres, if serum creatinine was greater than $200 \mu \mathrm{mol} / 1$, or if hepatic failure resulted in a prothrombin ratio greater than 1.8 or in hepatic encephalopathy. Patients who had been admitted to the trial previously were not included a second time. For administrative reasons a proportion of patients who fulfilled inclusion criteria was not included. This was not determined on clinical grounds and no patient was excluded after randomisation.

\section{Assignment}

After the decision had been made to feed the patient parenterally, informed consent was sought from the patient or, if this was not possible, from a relative. A standard written form of words, agreed with the ethics committee, was used with additional verbal explanations as required, and written consent obtained. After consent the patient was randomised to receive or not to receive $20 \mathrm{~g}$ glutamine included in the parenteral feed as part of the overall estimated nitrogen requirement, to be continued for the duration of any parenteral feeding for that hospital admission. Randomisation was carried out in the pharmacy using sealed envelopes such that the assigning pharmacist could not know the group to which the patient would be randomised prior to the decision. Randomisation was restricted in blocks of 10 to ensure that approximately equal numbers of patients received or did not receive glutamine at any time point during the trial.

\section{Masking}

The patients' feeds were delivered daily to the patients' ward from the pharmacy. Glutamine and non-glutamine containing feeds were labelled identically and there was no means by which the attending nutrition team or other clinical carers could know to which group the patient was randomised. The masking was revealed to the principal investigators after 50 patients were included in the trial and again at the end of the study when 170 patients had been randomised. The decision to discharge patients from hospital was made independently of the nutrition team administering the trial, usually a considerable time after cessation of parenteral feeding.

\section{Regimens}

The patients' energy and nitrogen requirements were determined using a standard computer protocol ${ }^{12}$ for all patients in the trial. This approach has been validated in a survey of its use conducted in 197 patients (not the same as the previously cited audit) over the age of 16 to achieve appropriate weight gain (mean 0.43 $\mathrm{kg} /$ week) in patients in whom this is desired, and good weight stability $(+0.06 \mathrm{~kg} /$ week) in those for whom this is the clinical goal. ${ }^{13}{ }^{14} \mathrm{In}$ order to ensure that a minimum requirement of essential amino acids was supplied to both treatment groups, and bearing in mind that 20 g glutamine represents $3.8 \mathrm{~g}$ nitrogen, we set a minimum total nitrogen intake of $11 \mathrm{~g}$ nitrogen for all trial patients. All feeds were prescribed by the nutrition team and contained a full complement of trace elements (Additrace 10 $\mathrm{ml}$ ), vitamins (Solivito $\mathrm{N}$ with Vitlipid, or Cernevit); calcium, phosphate, magnesium, potassium, and sodium requirements were individualised, closely so for the latter electrolytes, and volume determined according to the patients' needs and losses. The glutamine containing parenteral feeds provided $20 \mathrm{~g}$ glutamine, with the remainder of the estimated nitrogen requirement supplied as Eloamin. The nitrogen source of the non-glutamine containing feeds varied between Vamin 14, Vamin 18, Intrafusin, and Synthamin.

PRIMARY OUTCOME MEASURES AND SAMPLE SIZE CALCULATION

Mortality

The pilot audit showed a control mortality of $68 / 192$ patients $(35 \%)$. In order to show a halving of this mortality at the $5 \%$ level of significance with a power of $80 \%$, a total of 180 patients, 90 in each group was required. This postulate of an approximate halving of mortality was thought to be realistic at planned review of the trial after inclusion of the first 49 patients when control mortality was $36 \%$ $(9 / 25)$ and that of glutamine supplemented patients $16.7 \%$ (4/24). The target of including 180 patients was therefore set. In the event practical staffing issues determined, prior to unmasking, that the trial was terminated after inclusion of 170 patients. The mortality and length of stay among those patients treated by the nutrition team during the period of the trial, but not randomised, was also determined.

Length of hospital stay

Morbidity was assessed using length of hospital stay as a surrogate. If control length of stay was 44 (34) days a trial including 170 patients has the power to show a mean reduction of just under 10 days, $\mathrm{p}=0.05,1-\beta=0.8$.

SECONDARY OUTCOME MEASURES

Comparison of cause of hospital death between groups

The records of death certificates given by the clinicians in charge of the case (independent of the nutrition team) of those that died during the period of their trial-index hospital stay were used and cause of death recorded and grouped before the observer knew the assigned treatment group. Two systems of grouping were used and the results from both given.

\section{Septic complication rates}

At the completion of the trial a computer search was made for each patient under microbiological investigation, by an investigator unaware of the group to which the patient was allocated. Positive cultures were recorded. Analysis was: (a) by comparing the groups for the total number of positive cultures; and $(b)$ by considering a patient as positive if they had any single positive clinically relevant culture, and negative if not.

Perceived morbidity/"quality of life" scores

Patients were asked to score their mood, sleep, energy, appetite, pain, and mobilisation on a 10 point scale with $0=$ "perfect" and $10=$ "as bad 


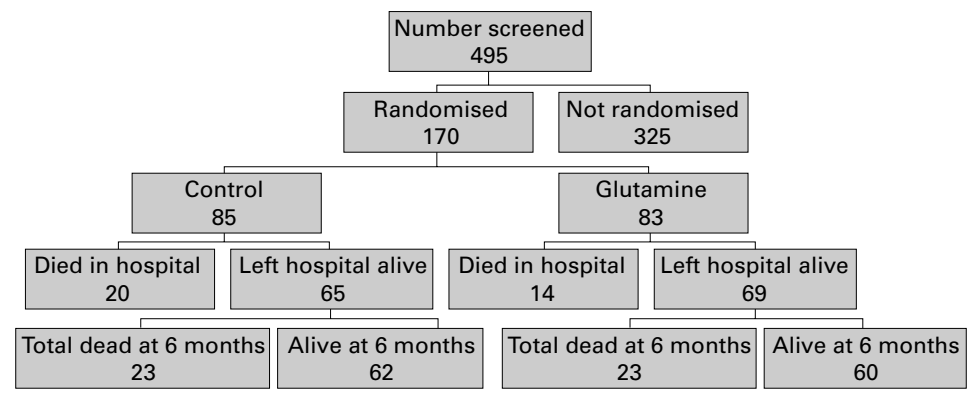

Figure 1 The structure of the trial showing the numbers of patients screened, randomised, dying, and surviving.

as it can be", at entry into the trial and again when parenteral feeding ceased.

Plasma amino acid concentrations

Plasma amino acid concentration was measured prior to starting parenteral nutrition and again just prior to stopping the parenteral feed, in 41 patients using an HPLC technique. ${ }^{1516}$ Blood was kept on ice until plasma was separated within a maximum of 30 minutes, and samples were stored at $-70^{\circ} \mathrm{C}$ until analysis.

\section{Results}

PARTICIPANT FLOW AND FOLLOW UP

Figure 1 shows the total number of patients treated with parenteral nutrition by the nutrition team during the period of the trial, the number randomised, and the principal mortality data. A total of 495 patients was treated with parenteral feeding during the period, of whom 170 were included in the trial and 325 not included. Of the 170 included, two consented but were not randomised as the

Table 1 Comparison of the two treatment groups

\begin{tabular}{lll}
\hline & Control & Glutamine \\
\hline $\begin{array}{l}\text { Total number } \\
\text { Male/female }\end{array}$ & 85 & 83 \\
$\begin{array}{l}\text { Mean (SD) age } \\
\text { Median (IQ range) BMI }\left(\mathrm{kg} / \mathrm{m}^{2}\right)\end{array}$ & $52 / 33$ & $49 / 34$ \\
$\begin{array}{l}\text { Median (IQ range) duration of parenteral feeding } \\
\quad \text { (days) }\end{array}$ & $22(21-24)$ & $48(17)$ \\
$\begin{array}{l}\text { Median (IQ range) number of parenteral feed bags } \\
\text { prescribed }\end{array}$ & $7(4-12)$ & $23(21-24)$ \\
$\begin{array}{l}\text { Mean (SD) energy intake }(\mathrm{MJ} / \mathrm{kg}) \\
\text { Median (IQ range) parenteral energy intake }\end{array}$ & $0.13(0.03)$ & $8(4-15)$ \\
$\quad(\mathrm{MJ} / 24$ hours) & $8.36(7.52-9.19)$ & $8.13(0.03)$ \\
$\begin{array}{l}\text { Mean (SD) nitrogen intake }(\mathrm{g} / \mathrm{kg}) \\
\text { Median (IQ range) of each patient's mean }\end{array} \quad 0.20(0.07)$ & $0.21(0.07)$ \\
$\quad$ parenteral N intake $(\mathrm{g} / 24$ hours) & $14(11-14)$ & $14(11-14)$ \\
$\begin{array}{l}\text { Mean (SD) body temperature }\left({ }^{\circ} \mathrm{C}\right) \\
\text { Mean (SD) plasma albumin at entry (g/l) }\end{array}$ & $37.2(0.42)$ & $37.1(0.37)$ \\
\hline
\end{tabular}

$\mathrm{IQ}$, interquartile; SD, standard deviation; BMI, body mass index

Table 2 Comparison of the two groups by the directorate under which the patient was treated

\begin{tabular}{|c|c|c|c|c|}
\hline & \multicolumn{2}{|c|}{ Control } & \multicolumn{2}{|c|}{ Glutamine } \\
\hline & Total & Died in hospital & Total & Died in hospital \\
\hline Haematology & 32 & 7 & 30 & 3 \\
\hline Surgery & 38 & 10 & 43 & 10 \\
\hline Non-surgical ICU & 3 & - & 1 & - \\
\hline Gynaecology & 0 & - & 1 & - \\
\hline Medicine & 12 & 3 & 8 & 1 \\
\hline Total & 85 & 20 & 83 & 14 \\
\hline Pooled ICU & 25 & 9 & 17 & 10 \\
\hline
\end{tabular}

Pooled intensive care unit (ICU) patients are those from any directorate who received intensive care during the index admission. There is no statistically significant difference between the groups. decision to feed them parenterally was rescinded, leaving 168 in the trial; 83 were randomised to receive glutamine supplementation, and 85 to the control regimen.

COMPARISON OF THE TWO GROUPS

The mean (range) age of the 325 patients not included was 53.2 (14-86) years, with 203 $(62 \%)$ of these patients male; $26 \%$ of these were managed in the intensive care unit. Table 1 shows the randomised groups by age, sex, height, and weight, and gives the median (interquartile range) energy and nitrogen intake by group. It can be seen that the length of parenteral feeding as judged by the number of bags of feed prescribed, and the pretreatment serum albumin, were very similar. Table 2 shows the hospital directorates under whose principal care the patients were treated. Patients could be classified according to whether they were treated on the intensive care unit or not during their admission and this is shown separately. It can be seen that in all respects randomisation produced two similar groups.

\section{ANALYSIS}

Figure 1 shows the mortality data for the trial patients. Hospital mortality in the glutamine group was $16.9 \%$ compared with $24 \%$ in the control group. There was no significant difference in this respect between the trial groups $(p=0.28)$, with six month mortality nearly identical. Mortality during the hospital admission for the control group was significantly lower $(p=0.01)$ than the $35 \%$ mortality observed in the audit. Mortality in the glutamine group was $16.9 \%$ which is also significantly different from the audit mortality rate $(p=0.002)$. A mortality of $37.6 \%$ was observed among the patients not randomised. Of the 69 patients who left hospital alive after receiving glutamine, nine were dead within six months, compared with three of the 62 patients who left hospital alive after control treatment (NS); by six months the outcomes in the two treatment groups were the same with a total of 23 dying in each group.

Median (interquartile range) length of stay in the audit was 36 (22-55) days and 32.5 (19$60)$ days for the non-randomised patients, compared with the median of 35 (25-55) days for the control group and 32 (23-52) days for the glutamine group. Length of stay data were skewed even after log transformation ( $\mathrm{pr}>\mathrm{Z}$ 0.03) so Mann-Whitney testing was used; this showed that length of stay was not significantly different between control and glutamine groups $(p=0.23)$.

Table 3 presents the length of stay data for the three principal directorates in which patients were managed, together with the pooled data for patients from any directorate who spent some time on the intensive care unit. Glutamine supplementation was associated with a borderline significant reduction in length of stay among the surgical patients $(p<0.03)$ but this was not true for any other of the clinical directorates nor for the patients 
Table 3 Length of stay (days) in the three directorates with more than five patients included and in the pooled intensive care unit (ICU) patients

\begin{tabular}{llllll}
\hline & \multicolumn{2}{l}{ Control } & & \multicolumn{2}{l}{ Glutamine } \\
\cline { 2 - 3 } \cline { 5 - 6 } \cline { 5 - 6 } & Median & Interquartile range & & Median & Interquartile range \\
\hline Haematology & 32 & $24-43$ & & 33 & $26-43$ \\
Surgery & 45 & $29-81$ & & 30 & $19-54$ \\
Medicine & 32 & $24-86$ & & 42 & $21-75$ \\
Pooled ICU & 37.5 & $29-79$ & & 45 & $16-85$ \\
\hline
\end{tabular}

Table 4 Total number of patients with positive microbiological cultures

\begin{tabular}{llll}
\hline $\begin{array}{l}\text { Infective complications: number of } \\
\text { positive cultures per patient }\end{array}$ & Control & Glutamine & Total \\
\hline 0 & 37 & 35 & 72 \\
1 & 16 & 16 & 32 \\
2 & 10 & 8 & 18 \\
3 & 6 & 6 & 12 \\
4 & 3 & 5 & 8 \\
5 & 1 & 1 & 2 \\
6 & 2 & 0 & 2 \\
10 & 0 & 1 & 1 \\
Total & 75 & 72 & 147
\end{tabular}

Numbers represent numbers of patients. There is no statistically significant difference between the two groups. pairs, improved ( $\mathrm{p}<0.0001$ for each), but there was no statistical difference in the improvement seen between the two treatment groups for any of the modalities.

\section{PLASMA GLUTAMINE CONCENTRATIONS}

Twenty four patients on standard feeds and 17 on glutamine containing feeds contributed paired plasma samples before and at the completion of parenteral feeding. No differences were seen between pretreatment or posttreatment concentrations of the amino acids measured; therefore only data for glutamine concentrations are presented. Prefeeding glutamine concentrations were 323 (SD 12) and 394 (165) $\mu \mathrm{mol} / 1$ in standard and glutamine groups, respectively, and completion concentrations were 342 (186) and 470 (229) $\mu \mathrm{mol} / 1$, respectively. No statistical difference was seen between either prefeeding and completion results or between groups before treatment or at completion.

(from all directorates) treated on the intensive care unit.

\section{SEPTIC COMPLICATIONS}

The infectious complication rates were closely similar for the two treatment groups with 38 patients in the control group and 37 in the glutamine group having at least one positive culture (table 4 ).

\section{CAUSE OF DEATH}

The certified cause of death was reviewed in all cases and was analysed by two methods only (table 5). In the first (method A), cause of death (which included sepsis, pneumonia, and multiple organ failure) was used rather than an underlying malignancy; in the second (method B), reanalysis was done with mortality related to haematological malignancy or other malignancy taking precedence over any terminal sepsis, pneumonia, or multiple organ failure. The overall differences between results obtained by the two methods were not significant although trends were observed towards fewer deaths from haematological malignancy and sepsis, pneumonia, or multiple organ failure on glutamine.

SUBJECTIVE MORBIDITY

All the modalities of morbidity/quality of life, individually assessed by Wilcoxon ranked

Table 5 Cause of death by group

\begin{tabular}{llllll}
\hline & 1 & 2 & 3 & 4 & Total \\
\hline $\begin{array}{lllll}\text { Method } A \\
\text { Control }\end{array}$ & 2 & 12 & 2 & 0 & 16 \\
Glutamine & 3 & 8 & 1 & 2 & 14 \\
Total & 5 & 20 & 3 & 2 & 30 \\
Method $B$ & & 3 & 6 & 0 & 16 \\
Control & 7 & 6 & 3 & 2 & 14 \\
Glutamine & 3 & 9 & 9 & 2 & 30 \\
Total & 10 & &
\end{tabular}

Method A: number of patients dying by cause and group. Death by infection (sepsis, pneumonia, and multiple organ failure) has been given precedence over any underlying malignancy. $1=$ ischaemia/infarction; $2=$ sepsis, pneumonia, and multiple organ failure; $3=$ cancer without $2 ; 4=$ road traffic accident $(\mathrm{p}=0.36)$.

Method B: number of patients dying by cause and group. Underlying malignancy has been given precedence over terminal sepsis, pneumonia, or multiple organ failure. 1 = haematological malignancy; $2=$ other malignancy; 3 = sepsis, pneumonia, or multiple organ failure without 1 or $2 ; 4=$ road traffic accident $(\mathrm{p}=0.14)$.

\section{Discussion}

Many studies over the past 10 years or so have suggested that intravascular and free muscle glutamine pools become depleted in response to stress, ${ }^{3}{ }^{17} 18$ and that these can be at least partially repleted by providing glutamine in doses such as those used in this trial, which exceed normal dietary or enteral feed intake by fourfold or more. ${ }^{519}$ Doses of $20 \mathrm{~g}$ compare with overall net daily glutamine turnover as judged by arteriovenous difference, ${ }^{5}$ but are severalfold less than the turnover of this amino acid as judged by isotope labelling studies. ${ }^{20-22}$ Surgical patients fed with glutamine supplemented parenteral nutrition have enhanced $\mathrm{T}$ lymphocyte DNA production compared with patients fed with standard parenteral feeds, ${ }^{4}$ and the mucosal integrity and absorptive function of the small intestine is better maintained than with standard parenteral feeds. ${ }^{67}$

This trial is the largest so far to test the potential overall clinical outcome benefits of supplementing parenteral feeds with glutamine. We used free glutamine rather than the dipeptide, but, bearing in mind the excellent metabolic availability of the dipeptide, ${ }^{3}$ can see no reason why our results could not be extrapolated to use of the dipeptide in equivalent doses. 
Table 6 Essential amino acid composition of feeds used

\begin{tabular}{|c|c|c|c|c|c|c|c|c|c|c|}
\hline & Ileu & Leu & Lys & Met & Phe & $T h r$ & $\operatorname{Trp}$ & Val & $\operatorname{Arg}$ & His \\
\hline Max RNI $55.5 \mathrm{~g} /$ day $\times$ preschool child & 1055 & 3663 & 3219 & & & 1887 & 611 & 1943 & 0 & 1055 \\
\hline Max RNI $55.5 \mathrm{~g} /$ day $\times$ adult & 888 & 1055 & 888 & & & 500 & 278 & 722 & 0 & 888 \\
\hline Max RNI $55.5 \mathrm{~g} /$ day $\times$ revised recommendation & 2109 & 3608 & 3885 & & & 1943 & 555 & 2220 & 0 & 0 \\
\hline Eloamin + Gln (14) & 2231 & 4016 & 4144 & 1211 & 2869 & 2678 & 956 & 3953 & 5100 & 3825 \\
\hline Eloamin + Gln (11) & 1575 & 2835 & 2925 & 855 & 2025 & 1890 & 675 & 2790 & 3600 & 2700 \\
\hline Synthamin & 4125 & 5019 & 3988 & 2750 & 3850 & 2888 & 1238 & 3988 & 7906 & 3300 \\
\hline Vamin $14 / 18$ & 3383 & 4758 & 5479 & 3383 & 4758 & 3383 & 1128 & 4434 & 6772 & 4111 \\
\hline Intrafusin 22 & 1547 & 2571 & 6841 & 2441 & 1849 & 2441 & 949 & 2124 & 6318 & 1581 \\
\hline Eloamin & 2406 & 4331 & 4469 & 1306 & 3094 & 2888 & 1031 & 4263 & 5500 & 4125 \\
\hline
\end{tabular}

Comparison of the indispensible amino acid constituents $(\mathrm{mg})$ of $11 \mathrm{~g} \mathrm{~N}$ of Synthamin, Vamin 14/18, Intrafusin 22, and Eloamin with the amino acid constituents of the reference nutrient intake (RNI) for protein of a male aged 19-50 years ( $55.5 \mathrm{~g} /$ day) when the protein conformed to three FAO/WHO patterns: that recommended for a preschool (growing) child; that recommended for an adult; and the 1991 revised recommendations for an adult. Further comparison is made with the Eloamin/ glutamine mixture when presented as an $11 \mathrm{~g} \mathrm{~N}$ or $14 \mathrm{~g} \mathrm{~N}$ feed. The Eloamin/glutamine mixtures both contain $20000 \mathrm{mg}$ glutamine; the other parenteral amino acid mixtures contain no glutamine.

PROTOCOL DEVIATIONS

Protocol deviations were not a problem in this study except that two patients consented who in practice subsequently were not fed parenterally; the decision not to provide them with parenteral nutrition was made before randomisation.

SPECIFIC INTERPRETATION OF STUDY INCLUDING SOURCES OF BIAS AND INTERNAL IMPRECISION Because we were using free glutamine rather than the dipeptide our feeds were pharmaceutically constrained by a need to use a volume of at least 2 litres. ${ }^{1}$ We did not feel confident to advise use of glutamine in the context of renal or hepatic failure (as defined) and the resulting exclusions will inevitably have excluded some of the sickest patients. These exclusions, combined with practical considerations resulted in our randomising 170 patients from a total of 495 screened. The extent that this affected overall results can be gauged by comparison of the trial control group with the unselected pretrial audit results and the overall mortality and length of stay observed in the patients screened but not randomised. Clearly our exclusion criteria had the effect of excluding some of the sickest patients from the trial. We excluded those needing less than 2 litres of feed per day because the pharmacy could not provide stable solutions containing glutamine below this volume. Hepatic failure patients were excluded because of the known relation of high cerebrospinal fluid glutamine concentrations and portasystemic encephalopathy. ${ }^{23}$ It is now known that an oral glutamine load worsens encephalopathy in cirrhotic patients. ${ }^{24}$ Renal failure patients were excluded because though it is well known that glutamine acts as a carrier for ammonia and as a source for urinary ammonium, very little is known about the effects of glutamine infusion on ammonia excretion or acid-base status in this context.

Table 6 shows the essential amino acid composition of the $11 \mathrm{~g}$ nitrogen and $14 \mathrm{~g}$ nitrogen glutamine feeds we used and can be compared with the input of indispensible amino acids which would be achieved, using standard feeds, by administering a reference nutrient intake (RNI) for protein for an adult of $55.5 \mathrm{~g}$ protein/day (8.9 g nitrogen) which is the highest of the RNIs for normal adults. It is inevitable that replacing some of the nitrogen proportionally reduces the content of the other amino acids - this was the price we had to pay for stipulating an isonitrogenous input between groups. An alternative trial design would have been to add another dispensible amino acid to the "standard" feeds to make up the nitrogen content equivalent to the glutamine. We rejected this approach because the standard feed would then have ceased to be an accurate reflection of what we normally do, not only because of the extra amino acid but also because of the extra nitrogen. Thus our trial should be viewed as a "pragmatic" test of the clinical strategy of glutamine supplementation.

\section{MORTALITY}

Although hospital mortality in the pretrial audit was $35 \%$ and mortality in the glutamine treated group was $16.9 \%$ (a significant difference), much of this reduction was probably the result of exclusion criteria applied before entry into the trial, as evidenced by the trial control mortality of $24 \%$, which itself was significantly different from the non-randomised patients and audit mortality. The remaining overall difference between the two trial groups could be explained on the basis of chance. There was no trend for reduction in mortality among surgical patients (who dominated the pooled intensive care admissions also), but there were statistically non-significant trends among haematology and medical patients; these trends have the potential for being very important clinically, suggesting a possible halving of mortality among these patients. There was, however, no trend for reduced mortality at six months. While there were only 24 medical patients, the haematology group included 62 patients, substantially more than the studies of Ziegler et $a l^{10}$ and Schloerb and Amare.

\section{LENGTH OF STAY}

Length of hospital stay provides an outcome measure which should reflect two important issues: morbidity and the cost of care. It may be confounded by mortality in so far as early hospital mortality may tend to reduce length of stay, thereby paradoxically suggesting improved outcome. In this study this was not a dominant problem, with length of stay among patients who died being similar to that among those who survived. Length of stay data in this group of patients were highly skewed so that even with log transformation they could not be represented by a normal distribution. We did 
not feel comfortable with the technique of exclusion of outliers in length of stay results used by Ziegler $e t a l^{10}$ and Schloerb and Amare ${ }^{9}$ and preferred to use non-parametric statistics. We saw no clear overall reduction in length of stay, though the trend of the trial was for a slight reduction when glutamine was used. This reduction was most notable among surgical patients but was not, despite the mortality trends, observed in the haematological patients in whom hospital stay is strongly protocol driven. Estimations in this Trust suggest that reduction in length of stay among surgical patients could be valued at $£ 100$ (\$160) per day (P McGinley, personal communication). If we assume from the trial results that a true 15 day reduction in length of stay among surgical patients is associated with a mean infusion period of eight days, with current costs for supplementation of glutamine using the free amino acid or its alanine dipeptide at around $£ 25$ (\$40) per day, our results suggest that the use of glutamine in the surgical patients had the potential to produce overall savings of around $£ 1000$ (\$1600) per patient. If the three day difference seen overall is taken as the best estimate of any length of stay advantage for glutamine, the treatment could not be regarded as cost effective, with costs of $£ 400$ (\$640) and savings of about $£ 300$ ( $\$ 480$ ).

OTHER OUTCOMES

Other trials have suggested that the use of glutamine may reduce infective complications which may tend to be increased in patients fed parenterally. This is a simple enough concept but one which presents enormous difficulties as far as a controlled trial is concerned, particularly when the trial, like this one, is conducted in different clinical units in the context of a hospital wide series of indications. To be valid data need to be gathered prospectively according to tight protocol driven definitions, which in turn need to be rigorously applied by the many attending clinicians. We realised from the outset that this would be impossible in practice in this trial and this was not attempted. However, we were able to analyse from computer records the number of clinically relevant positive cultures. For this purpose an observer unaware of the patient's group excluded positive routine nasal and skin cultures but included all other positive cultures for bacteria or yeasts. No difference was observed between the two groups in this respect, whether total numbers of cultures were considered or whether patients were regarded merely as having had or not had a positive culture. The amino acid concentrations did not differ between groups in this study, nor did they alter significantly during the course of the study. While all pretreatment concentrations were measured in the fasting state, those measured at the end of treatment, just before the parenteral feeding was stopped, varied in this respect and in some cases represent the effects of both parenteral and some oral or enteral intake. The data cannot therefore be directly compared with the study of Jensen et $a l^{25}$ conducted in 28 intensive care patients receiving glutamine enhanced enteral feeds over a 10 day period. In that study, while no difference was observed between glutamine supplemented and standard enterally fed patients at the end of the study, venous indispensible amino acids were increased on the control feed in comparison with the glutamine supplemented feed. The study of Hammarqvist et al, ${ }^{17}$ though it showed that free glutamine supplemented parenteral feeding could increase muscle free glutamine concentrations compared with standard parenteral feeds, also showed very little impact on plasma glutamine concentrations.

We made two (and only two) analyses of cause of death which are both given. In the first we analysed in such a way that "overwhelming infection", death from pneumonia or other infection combined or not with multiple organ failure had priority over other causes (particularly cancer) given. In the second the analysis was done after first separating out those patients dying of haematological or other malignancy. Neither showed statistically significant differences between the groups, though there were the previously noted possible reductions in mortality from haematological malignancy, and in death from "overwhelming infection", associated with glutamine administration.

Our technique for determining perceived morbidity was sufficiently sensitive to show highly statistically significant improvements among patients surviving to the end of their course of parenteral nutrition (and can be considered validated on this basis), but was able to detect no difference in any of the parameters recorded between the treatment groups by the end of the parenteral feeding course. This is in contrast with the study of Young et $a l^{26}$ conducted among those patients included in the study by Ziegler et al.

GENERAL INTERPRETATION OF THE DATA IN THE LIGHT OF THE TOTALITY OF THE AVAILABLE EVIDENCE

In this, the largest trial so far of glutamine supplementation of parenteral nutrition, we have been unable to confirm the clearcut positive findings of other smaller trials. We did not see a reduction in infective complications as shown in one trial conducted in patients with haematological malignancy ${ }^{10}$ (but not in another ${ }^{9}$ ), nor could we confirm the reductions in length of stay among such patients observed in both these studies. However our data suggest trends in mortality reduction among patients with haematological malignancy which may be important clinically, and is worthy of further study. There was some support for the reduction in length of stay among surgical patients observed in a small controlled trial by Furst's group ${ }^{27}$ from our subgroup analysis. Our data in 42 patients treated in the intensive care unit taken separately do not confirm the reductions in hospital mortality seen among intensive care patients by Griffiths et $a l^{11}$ nor the reduction in mortality at six months which they observed. This remains true if patients fed for less than five (or seven) days are excluded. 
However, it is of interest to note that the $29 \%$ reduction in mortality between glutamine supplemented and control groups in our complete study $(24 \%-17 \%=7 \%$, a reduction of $29 \%)$ is very similar to the $28 \%$ reduction in in-hospital mortality seen in that study.

While there are few statistically significant differences between the groups, there are some trends which may betray important clinical effects in reducing the high mortality among the very sick patients for whom parenteral feeding is reserved in our practice. In order to test further whether the observed reduction of $24 \%$ control mortality to $17 \%$ was real, a trial of 610 patients in each group would be required, setting the level of significance at 5\% and the power at $80 \%$. To confirm whether the observed difference in mortality in the haematological patients was real with a significance of $5 \%$ and a power of $80 \%$ would require 164 patients in each group. Under these circumstances we decided to publish these results now so that further consideration can be given to the possibility of a much larger trial which would require significant financial investment and a multicentre design. Until that is achieved glutamine supplementation cannot be recommended for routine use in parenteral feeding, even in units which, like ours, apply rigorous restriction on the use of intravenous feeding. Our own belief is that further trials should be conducted for more targeted indications, particularly in the context of support of leukaemia chemotherapy and complicated surgery where patients are particularly at risk of receiving total parenteral nutrition without any enteral intake.

Dr Powell-Tuck, Dr Jamieson, and Dr Obeid were supported by the Rank Prize Funds and Dr Bettany by the Martin Crabbe bequest. We gratefully acknowledge a grant from Fresenius Ltd UK which enabled the completion of the study. Dr G Hardy, Oxford Nutrition Ltd, supplied the glutamine and Eloamin a reduced cost. Dr Lindy Castell, Oxford University, was involved in early glutamine measurements. Ms Fawcett was employed by the Royal Hospital Trust as part of the Rank Department of Human Nutrition at the time she was involved with the trial. She was subsequently employed by Fresenius Ltd, after which she had no further involvement in the trial. Ms Vicky Eades is thanked for pharmaceutical help. We thank also Ms Joan Morris and Dr Filomena Pereira (Wolfson Institute, St BartholomDentistry) who provided statistical advice in the planning and analysis of the study.

1 Khan K, Hardy G, McElroy B, et al. The stability of L-glutamine in total parenteral nutrition solutions. Clin Nutr 1991;10:193-8.

2 Powell-Tuck J. Glutamine supplementation in artificial nutritional support. Lancet 1997;350:534.

3 Furst P, Pogan K, Stehle P. Glutamine dipeptides in clinical nutrition. Nutrition 1997;13:731-7.

4 O'Riordain MG, Fearon KC, Ross JA, et al. Glutaminesupplemented total parenteral nutrition enhances
T-lymphocyte response in surgical patients undergoing colorectal resection. Ann Surg 1994;220:212-21.

5 Elia M, Lunn PG. The use of glutamine in the treatment of gastrointestinal disease in man. Nutrition 1997;13:743-7.

6 Tremel H, Kienle B, Weilemann LS, et al. Glutamine dipeptide-supplemented parenteral nutrition maintains intestinal function in the critically ill. Gastroenterology 1994;107:1595-601.

7 van der Hulst RWJ, van Kreel BK, von Meyenfeldt MHF, et al. Glutamine and the preservation of gut integrity. Lancet 1993;341:1363-5.

8 Houdijk APJ, Rijnsburger ER, Jansen J, et al. Randomised trial of glutamine-enriched enteral nutrition on infectious mortality in patients with multiple trauma. Lancet 1998; 352:772-6.

9 Schloerb P, Amare M. Total parenteral nutriton with glutamine in bone marrow transplantation and other clinical applications (a randomized, double-blind study). $f$ Parent Enteral Nutr 1993;17:407-13.

10 Ziegler TR, Young LS, Benfell K, et al. Clinical and metabolic efficacy of glutamine-supplemented parenteral nutrition after bone marrow transplantation. A randomized, double-blind, controlled study. Ann Intern Med 1992;116:821-8.

11 Griffiths RD, Jones C, Palmer TE. Six-month outcome of critically ill patients given glutamine-supplemented critically ill patients given glutamine-supple
parenteral nutrition. Nutrition 1997;13:295-302.

12 Colley CM, Fleck A, Howard JP. Pocket computer: a new aid to nutritional support. $B M \mathcal{F} 1985 ; 290: 1403-6$.

13 Glynn MJ, Powell-Tuck J, Fleck A. Individualised prescribing of parenteral nutriton allows weight maintenance or weight gain as required. Clin Nutr 1988;7(suppl 1):o10.

14 Glynn MJ. The implications of insulin and protein metabolism for nutritional support. MD thesis, University of Cambridge, 1995.

15 Mann G, Smith S, Norman P, et al. Fasting, refeeding and diabetes modulate free amino acid concentrations in the rat exocrine pancreas: role of transstimulation in amino acid exflux. Pancreas 1988;3(suppl 1):67-76.

16 Cunliffe A, Obeid O, Powell-Tuck J. Post-prandial changes in measures of fatigue: effect of a mixed or a pure carbohydrate or pure fat meal. Eur F Clin Nutr 1997;51:831-8.

17 Hammarqvist F, Wernerman J, Ali R, et al. Addition of glutamine to total parenteral nutrition after elective abdominal surgery spares free glutamine in muscle, counteracts the fall in muscle protein synthesis, and improves nitrogen balance. Ann Surg 1989;209:455-61.

18 Stehle P, Zander J, Mertes N, et al. Effect of parentera glutamine supplements on muscle glutamine loss and
nitrogen balance after major surgery. Lancet 1989;i:231-3.

19 Kuhn KS, Stehle P, Furst P. Glutamine content of protein and peptide-based enteral products. F Parent Enteral Nutr 1996;20:292-5.

20 Darmaun D, Matthews DE, Bier DM. Glutamine and glutamate kinetics in humans. Am f Physiol 1986;251: E117-26.

21 Darmaun D, Matthews DE, Bier D. Physiological hypercortisolemia increases proteolysis, glutamine and alanine production. Am f Physiol 1988;255:E366-73.

22 Waterlow JC, Jackson AA, Golden MHN, et al. The effect of acidosis on the labelling of urinary ammonia during infusion of [amide-15N] glutamine in human subjects. $\mathrm{Br} \mathcal{F}$ Nutr 1994;72:83-92.

23 Hawkins RA, Jessy J, Mans AM, et al. Effect of reducing brain glutamine synthesis on metabolic symptoms of hepatic encephalopathy. $\mathcal{F}$ Neurochem 1993;60:1000-6.

24 Oppong KN, Al-Mardini $\mathrm{H}$, Thick M, et al. Oral glutamine challenge in cirrhotics pre- and post-liver transplantation: a psychometric and analyzed EEG study. Hepatology 1997; 26:870-6.

25 Jensen GL, Miller RH, Talabiska DG, et al. A double-blind, prospective, randomized study of glutamine-enriched compared with standard peptide-based feeding in critically ill patients. Am f Clin Nutr 1996;64:615-21.

26 Young LS, Bye R, Scheltinga M, et al. Patients receiving glutamine-supplemented intravenous feedings report an improvement in mood. F Parent Enteral Nutr 1993;17:422-

27 Morlion BJ, Stehle P, Wachtler P, et al. Total parenteral nutrition with glutamine dipeptide after major abdominal surgery: a randomized, double-blind, controlled study. Ann Surg 1998;227:302-8. 